\title{
One Day in the Life of a National Twittersphere
}

\author{
Axel Bruns ${ }^{I} \&$ Brenda Moon ${ }^{I I}$ \\ I Digital Media Research Centre, Queensland University of Technology, Brisbane, Australia, \\ a.bruns@qut.edu.au \\ II Digital Media Research Centre, Queensland University of Technology, Brisbane, Australia, \\ brenda.moon@qut.edu.au
}

\begin{abstract}
Previous research into social media platforms has often focused on the exceptional: key moments in politics, sports or crisis communication. For Twitter, it has usually centred on hashtags or keywords. Routine and everyday social media practices remain underexamined as a result; the literature has overrepresented the loudest voices: those users who contribute actively to popular hashtags. This article addresses this imbalance by exploring in depth the day-to-day patterns of activity within the Australian Twittersphere for a 24-hour period in March 2017. We focus especially on the previously less visible everyday social media practices that this shift in perspective reveals. This provides critical new insights into where, and how, to look for evidence of onlife traces in a systematic way.
\end{abstract}

Keywords: social media, Twitter, Australia, network analysis, phatic sharing

\section{Introduction}

Much of the existing research into the uses of social media has focused on the exceptional: key moments in politics (e.g. Larsson \& Moe, 2014; Papacharissi \& Blasiola, 2016), sports (e.g. Blaszka et al., 2012; Highfield, 2014) or crisis communication (e.g. Palen et al., 2010; Shaw et al., 2013). In Twitter research, because of the way in which the Twitter Application Programming Interface (API) privileges certain data-gathering approaches, such work has usually centred on hashtags or keywords (Burgess \& Bruns, 2015). This has produced many useful insights - as documented in the collection Hashtag publics (Rambukkana, 2015) - but covers only a subset of the platform's uses. Routine and everyday social media practices remain underexamined; the literature has overrepresented the loudest voices - those users who contribute actively to popular hashtags - and the communities of users that are already well known to researchers. Methodologically, it remains far more difficult to examine what is not already known: the ordinary, everyday, apparently unremarkable practices of the majority of Twitter users. 
Indeed, such mundane, everyday, ordinary uses (and users) have often been denigrated as banal and inconsequential: an early study of Twitter content by Pear Analytics infamously described some 41 per cent of all tweets as "pointless babble" of the "I am eating a sandwich now" variety (2009: 4-5). Such negative connotations, attached especially but not exclusively to supposed acts of oversharing, have persisted for social media uses that do not fit into acceptable categories of "meaningful" activity. They were eventually challenged, and platforms like Twitter were thereby "debanalised" (Rogers, 2014), yet even this gradual recognition more often proceeded by focusing on the non-mundane uses of social media that were seen as "meaningful" than by rescuing the mundane from its linkage with supposedly banal, pointless babble.

More recent contributions have highlighted the very meaningful roles that apparently "banal" uses of social media can play both for the users themselves and for their social networks. "'Banal' tweets serve as an important vehicle of self-affirmation", through identity creation and performance (Murthy, 2018: 32), and play a critical role in maintaining social relations with an "imagined audience" of followers (Marwick \& boyd, 2011). This focus on the mundane and everyday activities of ordinary users, across all digital media contexts, constitutes an important "shift in perspective" (Sandvik et al., 2016: 10): away from a focus on established, conventional producers of media content and towards the media experiences that users create for themselves, drawing both on such mainstream content and on their own materials. Social media have become a distinct "space of agency" (Sandvik et al., 2016: 15) that operates by its own logic (cf. van Dijck \& Poell, 2013) and has been domesticated more or less successfully into the everyday lives of its users (Haddon, 2016).

Addressing the theme of onlife traces, we suggest that it is especially in these mundane social media activities that the inextricable interweaving of online and offline lives should be most pronounced, if the thesis of an integrated "onlife" is correct (Simon \& Ess, 2015). While some of the extraordinary phenomena that are identified by Twitter hashtags also relate to notable offline events (such as protests, crises, media and sporting events), others are predominantly confined to the platforms themselves (including memes and other trending topics), and all of them are by definition exceptional. Only by looking beyond these exceptions and towards the digital traces of the everyday can we fully document how far online and offline are indeed blended into an ordinary, domestic onlife in the full sense. Finding consistent digital traces of an integrated onlife, even in users' non-exceptional social media practices, is considerably more significant than seeing them occur only in clearly unusual circumstances.

Methodologically, this desirable "shift in perspective" remains difficult. Mundane uses have been studied at a smaller scale through in-depth interviews, focus groups, media diaries and other forms of self-reporting, but large-scale observational data on the everyday practices of ordinary users are difficult to obtain: the data-gathering functionality of standard platform APIs inherently privileges the extraordinary. To oversimplify only slightly, the extraordinary can be filtered by searching for a small and well-known set of linguistic markers, such as keywords or hashtags. The mundane, by contrast, is all that remains after such content has been accounted for - and is not readily offered by the platforms' data interfaces. To capture the traces of onlife in the everyday activities of ordinary users, at scale, using native digital research methods, requires a more circuitous approach: first, we must solve the problem of how to identify these activities and distinguish the ordinary from the extraordinary. 
This article addresses this challenge by describing and implementing a framework for examining user activity patterns on Twitter well beyond limited hashtag collections, drawing on a comprehensive data set that tracks the public activities of all Australian Twitter accounts. For this cohort of 3.7 million accounts, we have already mapped the clustering patterns in follower/followee relationships (Bruns et al., 2017) that influence, arguably more than hashtags, how information flows between users. We have also identified the topical interests of these clusters and mapped participation in specific Twitter conversations across them.

We extend this work by exploring in depth the day-to-day patterns of activity within the Australian Twittersphere for a 24-hour period in March 2017. This provides new insights into how conversations between users unfold through the day and documents whether such interactions are guided by existing follower relationships, current hashtags or other factors. Our analysis shows which parts of the network are consistently active throughout the day, and which are triggered by new events; and which are more focused on publishing new content, on interpersonal conversation, or on news dissemination. This establishes a more complex picture of Twitter in Australia, beyond prominent hashtags, and, in the closing sections, we focus especially on the previously far less visible everyday practices that this shift in perspective reveals. We acknowledge that this does not solve the problem of extracting meaning from the onlife traces in such everyday, mundane activities by ordinary users, but we hope it provides scholars with critical new insights into where, and how, to look for such traces in systematic ways.

Overall, this article addresses the following research questions:

1. How do we shift the focus of Twitter research away from extraordinary events and towards mundane, everyday uses?

2. Does a whole-of-population data analysis enable previously overlooked everyday user practices to surface?

3. Do day-to-day activity patterns align with longer-term connection networks?

Our results provide new insights into the dynamics of Twitter engagement. They illuminate everyday users' utilization of Twitter and document the diversity of their communities. This is a reminder that the full story of social media in public and semi-public conversation can only be told if we look beyond the loudest voices. Finally, we highlight opportunities for further research.

\section{Data set}

Our analysis builds on Tracking Infrastructure for Social Media Analysis (TrISMA) (Bruns et al., 2016), a unique infrastructure that captures the public activities of an entire national Twittersphere. Filtering the global Twitter userbase for accounts that have recognizably Australian traits, TrISMA had identified 3.7 million Australian accounts by February 2016 and continuously tracks their public tweets; Bruns and colleagues (2017) provided more detail on this data set. For clarity, we note that this population includes individual users, group accounts, semi- and fully automated accounts (e.g. providing news updates) and various types of bots: these constitute a significant aspect of the lived experience of Twitter use, and we have therefore refrained from excluding them. 
For the present article, we selected a single 24-hour period of activity. We do not expect that period to represent longer-term patterns; rather, we are interested precisely in the particular peculiarities of an "ordinary" day in the Australian Twittersphere and in the analytical possibilities that such comparatively unfiltered data (in contrast to hashtag or keyword data sets) offer. That said, we avoided dates that we expected a priori to be dominated by known events; similarly, we selected a weekday rather than a weekend day, as longer-term tweeting patterns for Australia show considerably divergent and diminished activity on weekends.

Through an iterative process of reviewing the news coverage from candidate days and conducting some preliminary analyses of Twitter activity for these dates, we selected Wednesday 22 March 2017 for this study. Considering the diverse time zones across Australian states and territories and reflecting the location of major Australian population centres on the east coast, we used Australian Eastern Standard Time (AEST; UTC+10) to determine the start and end of this 24-hour time frame. We selected all public tweets posted by the 3.7 million Australian accounts known to TrISMA; we also extracted any hashtags,@mentions, retweets and URLs contained in the tweet texts. In total, this resulted in 1,294,569 tweets from 176,737 unique accounts actively posting to Twitter that day. The substantial discrepancy between the number of actively posting accounts on 22 March 2017 and the total number of Australian accounts known to TrISMA is unsurprising: first, many social media users engage in "listening" (Crawford, 2009) to public conversations rather than posting their own contributions; second, surveys have shown that many Australians use Twitter and similar services considerably less than once per day (Sensis, 2016). The TrISMA data set complements such reports with large-scale observational data from the platform itself.

\section{Previous work}

Using TrISMA data, Bruns and colleagues (2017) performed a network analysis of the follower/followee relationships between those 255,362 Australian Twitter accounts with at least 1,000 connections within the global Twittersphere, identifying the major, densely connected clusters of accounts. Through close reading of the profile information for leading accounts in each cluster, they determined these clusters' thematic focus. The validity of these labels was subsequently verified by Münch (forthcoming), using different methods.

Figure 1 (from Bruns et al., 2017: 6) shows the structure of this network, labelling the most prominent clusters; in the present article, we compare the patterns of interaction on 22 March 2017 with these much longer-term follower/followee relationships to assess whether everyday interactions are shaped by these networks or transcend the existing structures as users@mention or retweet accounts that they do not already follow. This is important not least in the context of the continuing debates about the influence of "echo chambers" (e.g. Sunstein, 2009) or "filter bubbles" (e.g. Pariser, 2011) on communication patterns. 
Figure 1. Follower-followee network among the best-connected accounts in the Australian Twittersphere

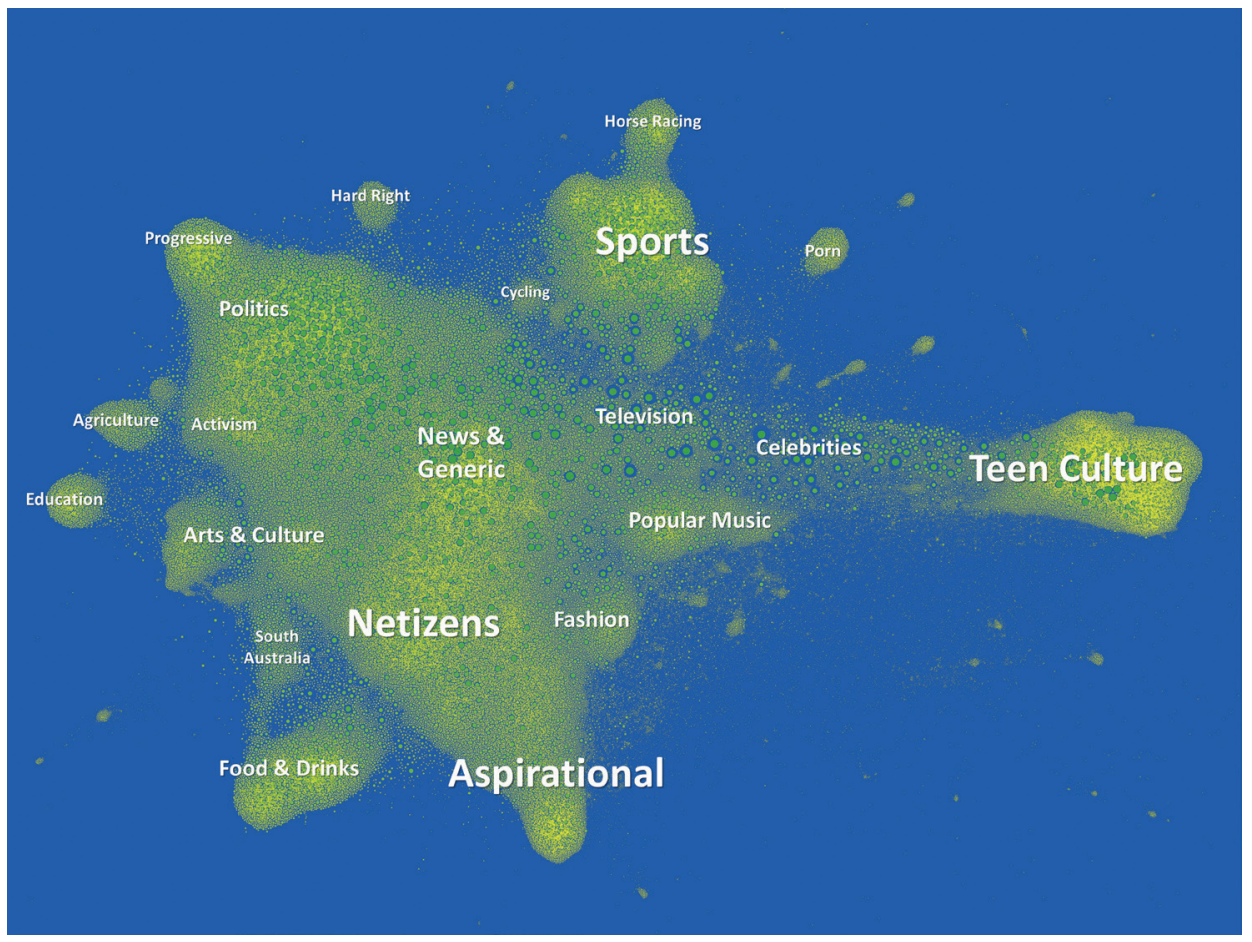

Comment: Selected clusters labelled according to their thematic focus (reproduced from Bruns et al., 2017)

\section{One day in the life of a national Twittersphere}

We turn first to an analysis of the overall activity levels across the Australian Twittersphere on 22 March 2017. This provides a unique insight into how Twitter is used within a specific national context and creates opportunities for comparison with other national Twitterspheres.

\section{Types of tweets}

Table 1 shows the overall distribution of activity across tweet types. The three major types of tweets (original tweets, @mentions and retweets) appear in relatively even proportions, with retweets being the most prominent. However, retweets were used by the smallest number of accounts: 50 per cent of Australian accounts posted at least one retweet compared with nearly 58 per cent posting an original tweet. This indicates an uneven distribution of tweeting styles across the account population, with retweets being more popular among smaller subsets of the entire Twittersphere. (The total percentages can add up to more than 100 per cent, since tweets can be both retweets and @mentions at the same time.) 
Table 1. Distribution of tweeting activity across different tweet types and styles, 22 March 2017

\begin{tabular}{lcccc}
\hline & Tweets & $\begin{array}{c}\text { Percentage of } \\
\text { tweets }\end{array}$ & $\begin{array}{c}\text { Unique } \\
\text { accounts }\end{array}$ & $\begin{array}{c}\text { Percentage of } \\
\text { accounts }\end{array}$ \\
\hline Total & $1,294,569$ & 100 & 176,737 & 100 \\
Original tweets & 427,537 & 33 & 101,785 & 58 \\
@mentions & 422,895 & 33 & 93,260 & 53 \\
Retweets & 555,003 & 43 & 88,752 & 50 \\
Hashtag & 301,405 & 23 & 72,672 & 41 \\
No hashtag & 993,164 & 77 & 104,065 & 59 \\
URL & 695,282 & 54 & 136,599 & 77 \\
No URL & 599,287 & 46 & 40,138 & 22 \\
Twitter.com URL & 365,995 & 28 & 84,263 & 48 \\
Non-Twitter.com URL & 369,177 & 29 & 90,044 & 51 \\
\hline
\end{tabular}

Source: TrISMA (Bruns et al., 2016).

Fewer than one-quarter of all tweets contained hashtags. This is remarkable given the considerable prominence of hashtag-centric studies and provides a major impetus for our study: if this finding is typical of usage practices in Australia and elsewhere, then hashtag studies provide insights into the self-selecting groups of hashtag users but ignore three-quarters of ordinary tweeting activity. Such a major limitation ought to be recognized in any presentation of such studies and addressed in their design.

Further, this significant imbalance between hashtagged and non-hashtagged tweets can arise only in part, if at all, from Twitter users' unfamiliarity with the concept of hashtags: although, overall, users chose to hashtag only one in four of their tweets, more than 41 per cent of accounts posted at least one hashtagged tweet during the day. Therefore, although many users are capable of using hashtags, they frequently choose not to do so. Overall, hashtags occurred in retweets $(28 \%)$ and original tweets $(26 \%)$ more often than@mentions (20\%): @mentions were thus used more frequently for small-scale conversations away from the enhanced visibility that hashtags facilitate. This supports the structure of micro-, meso- and macro-layers of communication on Twitter that Bruns and Moe (2014) proposed.

The day studied, 22 March 2017, exhibits a broadly balanced distribution of tweets with and without URLs: sharing links is a very common practice on Twitter. Indeed, more than three-quarters of all accounts posted at least one URL during the day. Almost two-thirds (62\%) of URL tweets pointed back to twitter.com, usually referencing previous tweets (through Twitter's “quoted tweet" functionality) or embedded photos and videos; a similar percentage (66\%) shared non-Twitter URLs, creating considerable circulation of genuinely outside content in the Australian Twittersphere. (Again, these percentages amount to more than $100 \%$, because many tweets included both Twitter and non-Twitter links within the same post, for instance combining external links with embedded images.) 


\section{Patterns through the day}

The overall tweeting activity varied over the day (Figure 2). Probably due to automated posts, differences in time zones and genuine night-time activity, the volumes never dropped below 28,000 tweets per hour, but the daytime activity was necessarily considerably higher, especially in the morning and evening. However, during the daytime, the typical number of unique accounts active per hour hovered consistently between 24,000 and 25,000; the daytime fluctuations in tweeting volume were therefore caused not by a net influx of new participants but by increased posting activity per account.

Figure 2a. Tweets and unique accounts per hour

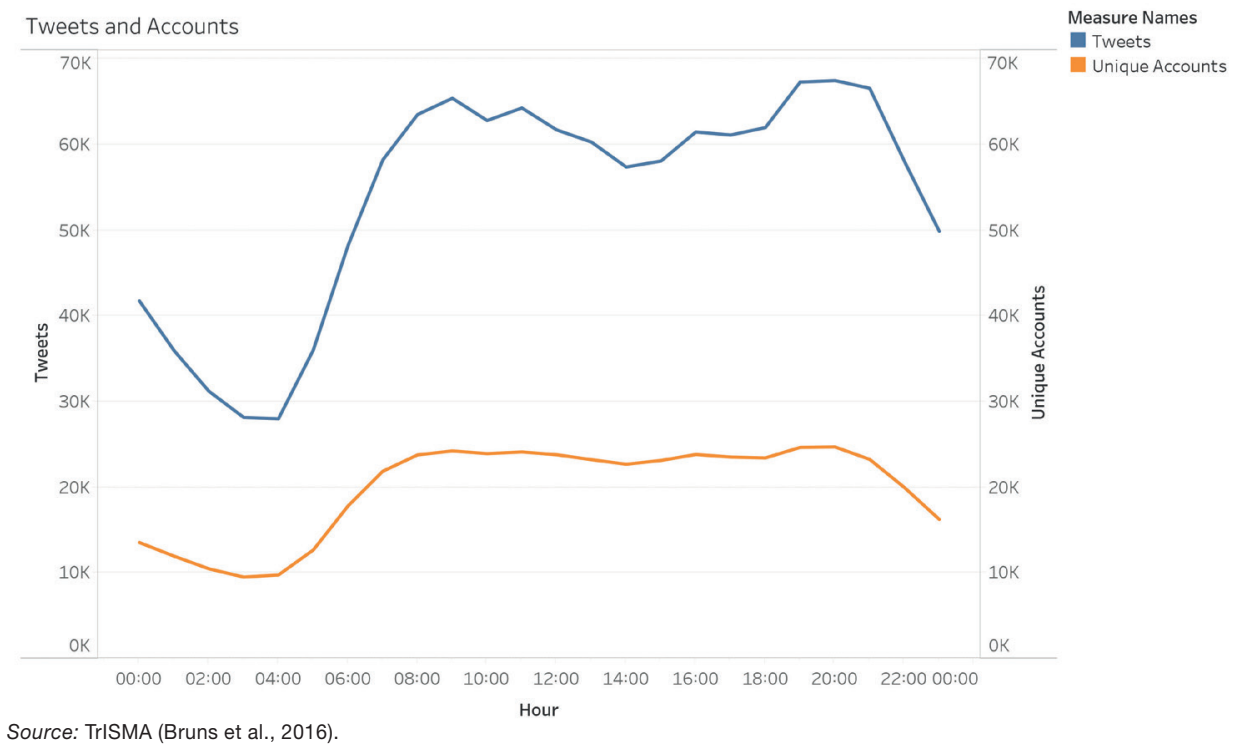

Figure 2b. Tweet types and hashtagged tweets per hour

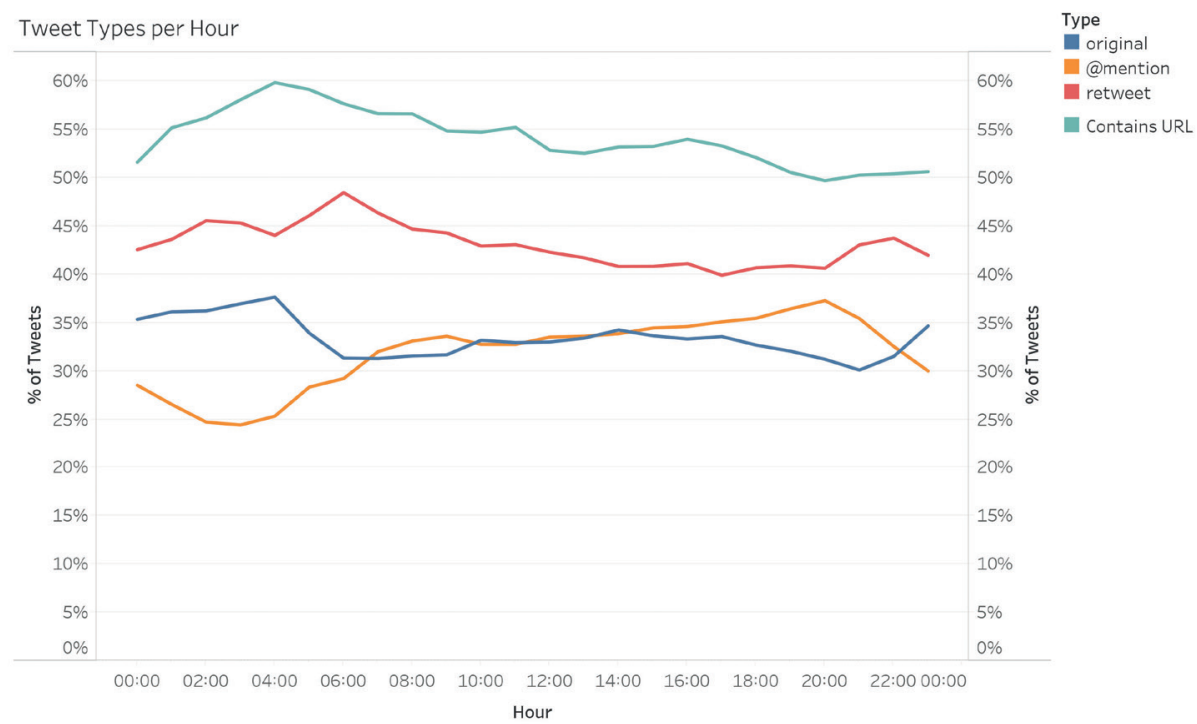

Source: TrISMA (Bruns et al., 2016). 
Such activity was distributed across tweeting types and styles. The fewer accounts active during the early hours posted more original tweets and fewer@mentions; clearly far fewer Australian accounts were active, reducing the potential for @mention engagement, but this remaining core of consistently active accounts might also include a greater proportion of accounts posting automated original messages and/or retweets. By contrast, after 06:00, the percentage of @mentions began to rise: to 33.61 per cent after 09:00 and then to 37.29 per cent after 19:00. Put simply, through the day, the Australian Twittersphere grew more discursive, especially after the conventional workday concluded.

Conversely, information sharing via retweets reached an early peak of 48.48 per cent of all tweets after 06:00 and then declined rather steadily; the late evening hours experienced a new increase (to 43.77 per cent after 22:00). This may relate especially to news reading and sharing; as users come online in the morning, they catch up with the news and retweet a proportion to their followers; similarly, as they end their day, they again engage especially with the latest news from Europe and North America, where the day is now underway. This is somewhat supported by the patterns of URL sharing: at 59.88 per cent, the hour after 04:00 accounted for the greatest percentage of URL tweets, declining to 49.72 per cent after 20:00. The increase in retweeting in the later evening did not coincide with more tweets containing URLs, however. Notably, tweets containing twitter.com URLs remained remarkably stable throughout the day, fluctuating only between 26.77 per cent and 29.82 per cent; any change in URL tweets was driven almost entirely by tweets linking to external content.

Hashtag use, meanwhile, largely followed the overall tweeting volumes. However, the nature of these hashtags changed markedly over the day. We identified 98,696 distinct hashtags, but their popularity followed a pronounced long-tail distribution: only 694 were used at least 100 times. Figure 3 shows the relative prominence of the 25 most common hashtags in each hour as a percentage of the 58,591 tweets that contained at least one such hashtag. (The percentages add up to more than $100 \%$ in each hour, as some tweets used two or more of these hashtags simultaneously.)

The early hours were dominated by hashtags that were relatively atypical for the remainder of the day. Prominent hashtags here included \#nowplaying, for instance in automated tweets that announced the tracks broadcast by radio stations in the Nova FM network; \#worldpoetryday, in genuine and spam posts that shared content related to World Poetry Day on 21 March; and \#wtl, almost exclusively in cryptic tweets from a now discontinued account. After 04:00, there was also a pronounced spike in \#free and/ or \#freedownload from a small number of accounts that promoted various e-books and software for search engine optimization, database management and big data. (These reappeared after 23:00.) Such patterns suggest that the small hours of the day are rife with bots and semi-automated accounts posting spam and algorithmically generated content.

After 06:00, the picture changed dramatically. From now until the late evening hours, political hashtags were prominent: these included the well-established hashtag for generic political discussion, \#auspol (cf. Highfield, 2013; McKinnon et al., 2016); \#18c and \#freedomofspeech for public debates about reducing prohibition against racial vilification in section 18c of Australia's Racial Discrimination Act, supposedly to strengthen Australians' right to "free speech" (McNamara, 2016); and \#qt, widely used by journalists, political staffers and other "political junkies" (Coleman, 2003) to live-tweet Question Time debates in the Australian federal parliament. The latter was 


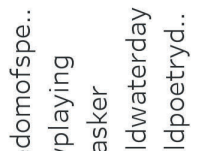

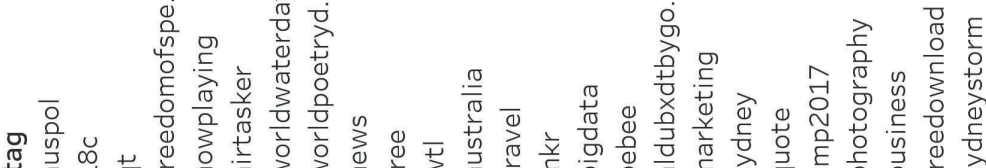

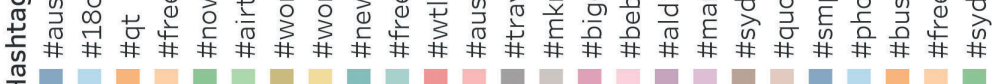

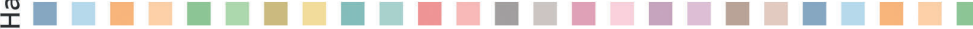
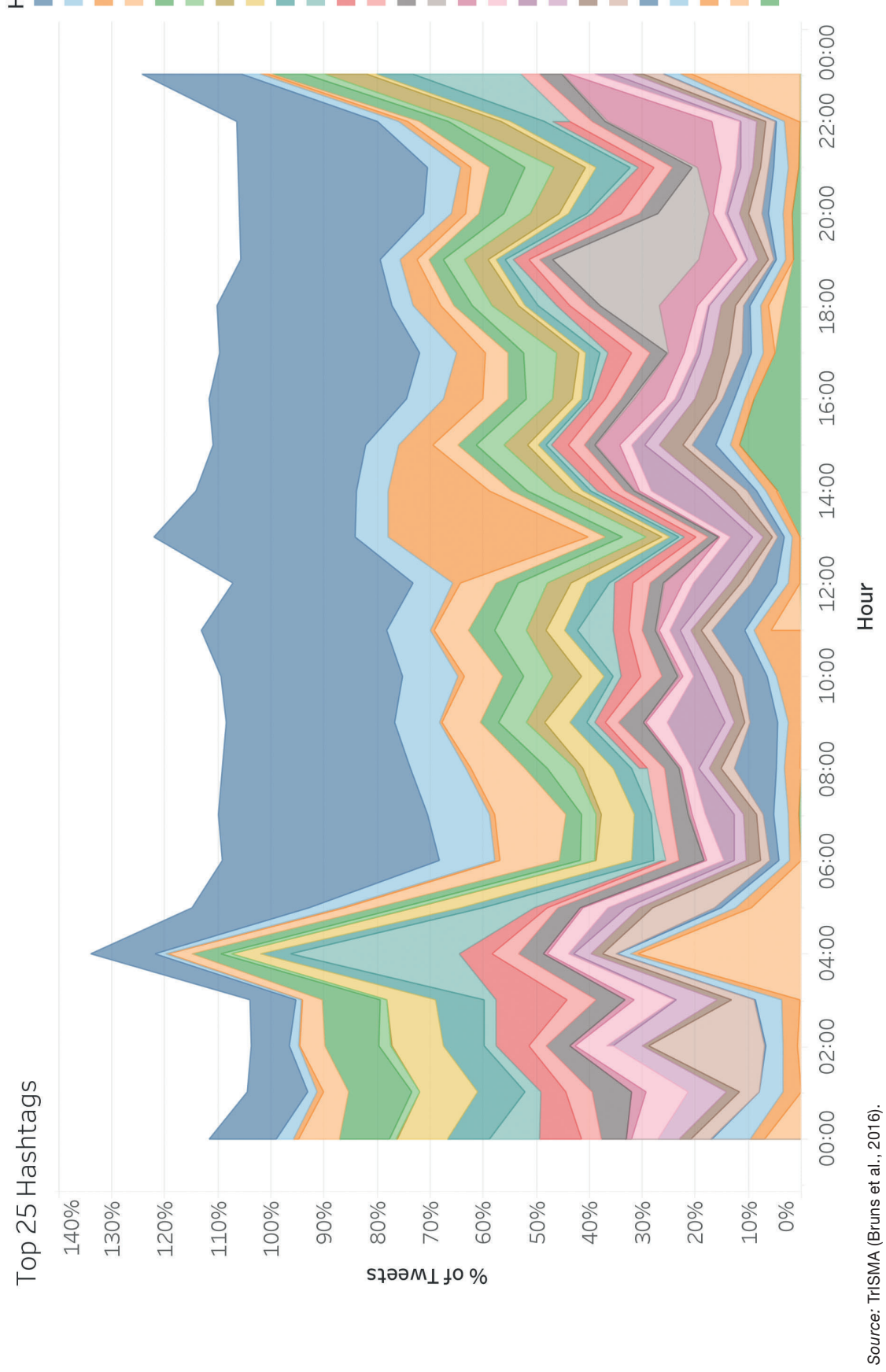
prominent from 13:00, as Question Time itself unfolded, with the subsequent discussion continuing throughout the remainder of the afternoon. Finally, \#smp2017, popular during the morning, tracked day two of the annual Science Meets Parliament event, facilitating engagement between scientists and federal politicians.

A handful of other hashtags appeared at more specific times. Between 14:00 and 18:00, \#sydneystorm waxed and waned as a major weather front moved across the greater Sydney area; such weather tweets are common in Australia, which is subject to major weather events, including cyclones, floods and bushfires. Between 18:00 and 21:00, the night's broadcast of the reality TV show My kitchen rules attracted a substantial number of participants to its "official" hashtag \#mkr, demonstrating the use of social media for second-screen engagement.

Finally, other terms represented more generic uses of hashtags. \#airtasker consisted almost exclusively of automated posts by city-specific accounts, such as @AirtaskerSYD,@AirtaskerMEL or@AirtaskerBNE, which promoted new casual job opportunities posted to the Airtasker platform. Meanwhile, hashtags such as \#news, \#australia, \#sydney, \#marketing or \#business were often used more to highlight the theme of a tweet than in the expectation that Twitter users will consistently track and engage in such hashtags. Their very generic nature made them appear in our top 25, but the hashtagged tweets were highly divergent and largely unrelated.

Notably, the four leading political hashtags accounted for nearly 44 per cent of the total number of tweets containing any of the top 25 hashtags. This result documents the considerable visibility of political debate in the Australian Twittersphere, both on this day and in relation to current debates and in the longer term. However, we stress here that the perceived prominence of political topics on Twitter in Australia stems largely from visibility rather than volume: the 25,592 tweets containing these four political hashtags represent a considerable subset of the 58,291 tweets containing any of the 25 most popular hashtags, or even of the 301,405 tweets containing any hashtags at all, but compare rather more poorly against the total of nearly 1.3 million tweets posted on 22 March 2017.

Australia's political junkies have long used standing hashtags such as \#auspol and \#qt and specific hashtags for the topics of the day to ensure the overall prominence of their issues within the national Twittersphere, yet users who neither follow these political accounts nor track the hashtags that they use may well remain disconnected from such political conversations. Ordinary, everyday, non-hashtagged conversations on Twitter should not be expected to mirror the themes of the leading hashtags: some such tweets may address political themes but perhaps deliberately eschew prominent political hashtags to avoid being drawn into public debates and controversies; others, meanwhile, will address far more niche and mundane topics that, in the eyes of their originators, do not require or deserve the enhanced visibility that a topical hashtag might afford. We must find a different approach to identifying and understanding such non-hashtagged tweets and their communicative functions.

\section{Network of interactions}

The topics of such non-hashtagged tweets might be determined (with difficulty and potential error, given the brevity of tweets) either through computational analysis or (for 
small purposive samples but not for the larger data set) through manual close reading. The scope of the present article and these considerable practical and methodological challenges did not permit us to determine the thematic focus of all non-hashtagged tweets; however, a network analysis of account interactions over the course of the day, against the backdrop of the follower map produced by Bruns and colleagues (2017), provided an opportunity to explore how and why Australian accounts engage with each other on Twitter.

We built this analysis on all @mentions and retweets, independent of whom they engage with; this means that the resultant data set also includes non-Australian Twitter accounts if they were mentioned or retweeted on 22 March 2017. Further, to focus on the most interactive accounts, we selected those accounts that posted or received at least10@mentions or retweets: that is, we filtered the network for nodes with a degree of 10 or more. This left 29,172 accounts (nodes in the network), connected by 400,520 @ mentions or retweets between 225,244 unique pairs of accounts (edges). Of these, 22,992 accounts $(79 \%)$ are included in the TrISMA data set; the remainder are either non-Australian accounts or Australian accounts created after the TrISMA data set was established in early 2016.

We visualized this network using the Force Atlas 2 algorithm in Gephi (Jacomy et al., 2014) and identified a number of distinct network clusters using the Louvain modularity algorithm, at modularity resolution 0.5 (Blondel et al., 2018). We further interpreted the thematic focus of these clusters by examining the most prominent nodes in each cluster and their tweets, manually assigning descriptive labels to these clusters. Accordingly, we reviewed the 100 accounts with the greatest in-degree (the greatest number of (a)mentions and retweets received) in each major cluster, focusing initially on their Twitter profile information. Usually, this revealed an obvious thematic focus: the prominent accounts in the Australian politics cluster, for example, included several current and former prime ministers, other senior politicians and leading political journalists. When this assessment of profiles did not produce clear patterns, we reviewed the tweets posted by and at these key accounts. This shed light especially on practices such as phatic sharing, which involve a diverse and heterogeneous group of accounts and are unified more by common behaviours than by a strong thematic focus.

We opted for this close reading approach, undertaken collaboratively between the authors, over formal manual coding, because the as-yet-unknown structure of the Twitter discussions required an inductive and even abductive approach (Dixon, 2012) that allowed meaningful descriptors to surface from the data. The thematic categories resulting from this close reading could now be used to inform further detailed coding, including the coding of data from other time periods, to establish precisely the balance between different themes and behaviours and trace how it changes over time, but this further analysis is not central to our present aims.

Figure 4 shows the result of this process. Table 2 presents the key activity metrics for selected clusters.

Notably, many clusters in the single-day interactions network, shown in Figure 4, correspond more or less directly to the clusters of the follower/followee network of 2016 (Bruns et al., 2017), shown in Figure 1. This indicates that such longer-term connections have a considerable influence on day-to-day interactions, even or especially if hashtags and other affordances are not used as facilitators of connection. We speculate that such 
Figure 4. Network of @mention and retweet interactions for accounts with degree $\geq 10$

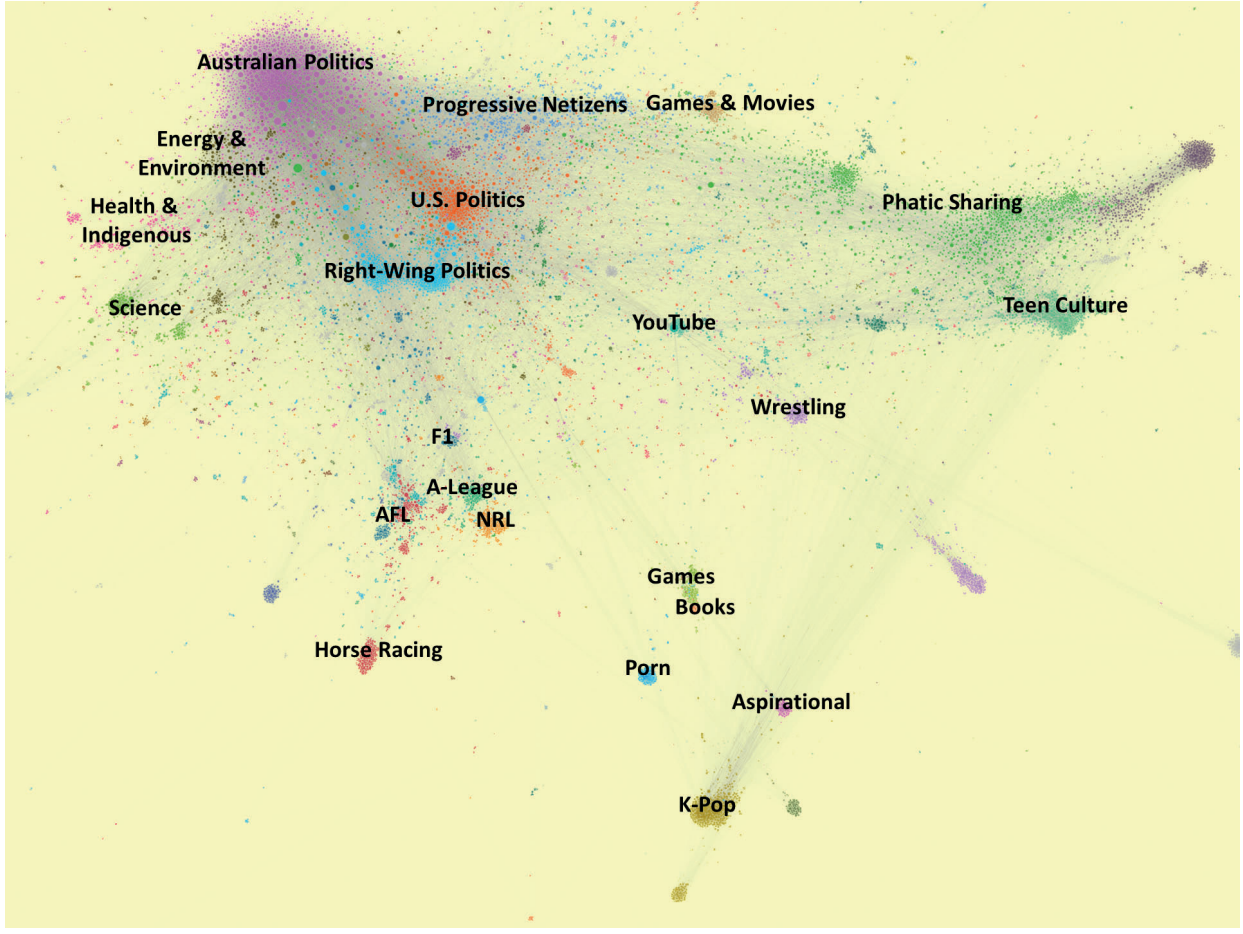

Comment: Force Atlas 2 algorithm, Louvain modularity detection (resolution 0.5). Selected clusters are labelled following qualitative interpretation.

Source: TrISMA (Bruns et al., 2016).

clusters would be present in interactions networks for other 24-hour periods, too. Other, often smaller clusters were unique to the interactions network: here, specific events of the day played a greater role in bringing Twitter accounts together in a shared interaction space, and hashtags are important in facilitating the formation of these temporary publics. Such practices are not mutually exclusive: during matchdays, for instance, the stable core of committed football fans in the AFL follower cluster may overlap to a considerable extent with a transient public of more casual sports fans using one or more of the dedicated match hashtags.

A prominent Australian politics cluster, containing 1,663 accounts, occupies the top left of the interactions network; its most mentioned accounts are leading Australian political figures, domestic journalists and news media. This cluster is separate from a cluster of 1,067 accounts that focuses on right-wing politics. Here, several conservative Australian news columnists and mainstream and fringe politicians are prominent, but the cluster also includes overseas (mainly US) political accounts, such as @realdonaldtrump and @POTUS, far-right and neo-fascist commentators and agitators and pro-Trump media from WikiLeaks to Fox News. This ideologically unified cluster, in turn, is separate from a cluster of 941 accounts that addresses general US politics and contains mainstream media accounts such as the New York Times, The Guardian and CNN, as well as key journalists, politicians and celebrities from the US. These clusters contain many non-Australian accounts that are not part of the TrISMA data set and appear here through@mentions and retweets; we therefore interpret these clusters as indicating the considerable attention 
paid to US political developments, only two months after Donald Trump's inauguration, by both mainstream and far-right Australian Twitter users. Located above them, in turn, is a cluster of progressive netizens: 963 institutional and individual accounts from media and technology fields that largely represent left-of-centre political views.

In these clusters, \#auspol is the most prominent hashtag; in Australian politics, the other major political hashtags are also used regularly, while in US politics, \#trumprussia has already emerged, and right-wing politics uses domestic and foreign hashtags, such as \#18c and \#MAGA. Hashtag use varies considerably, however: some 35 per cent of tweets by Australian politics accounts contained a hashtag, but only 10 per cent of tweets by progressive netizens. With 37 per cent, the latter group also accounts for few tweets containing URLs compared with between 47 per cent and 56 per cent in the other major clusters. By contrast, progressive netizens used considerably more@mentions and fewer retweets than other major political groups; retweets were particularly prominent in Australian politics (71\%) and US politics (69\%). This indicates sharply differing uses of Twitter's communicative affordances: in mainstream political clusters, information sharing and amplification via retweets is common, but ideologically slanted clusters emphasize discussion with (or about) others inside and outside their communities through@mentions.

Table 2. Key metrics for selected clusters in the interaction network

\begin{tabular}{|c|c|c|c|c|c|c|c|}
\hline Cluster & $\begin{array}{c}\text { Active } \\
\text { accounts }\end{array}$ & Tweets & $\begin{array}{l}\text { Hash- } \\
\text { tagged }\end{array}$ & Original & $\begin{array}{l}\text { @men- } \\
\text { tions }\end{array}$ & Retweets & $\begin{array}{l}\text { URL } \\
\text { tweets }\end{array}$ \\
\hline Phatic sharing & 2,440 & 88,912 & $5 \%$ & $23 \%$ & $25 \%$ & $56 \%$ & $48 \%$ \\
\hline Australian politics & 1,663 & 67,493 & $35 \%$ & $13 \%$ & $37 \%$ & $71 \%$ & $47 \%$ \\
\hline $\begin{array}{l}\text { Right-wing } \\
\text { politics }\end{array}$ & 1,067 & 38,023 & $23 \%$ & $14 \%$ & $43 \%$ & $57 \%$ & $48 \%$ \\
\hline $\begin{array}{l}\text { Progressive } \\
\text { netizens }\end{array}$ & 963 & 24,844 & $10 \%$ & $23 \%$ & $51 \%$ & $33 \%$ & $37 \%$ \\
\hline US politics & 941 & 34,001 & $22 \%$ & $12 \%$ & $32 \%$ & $69 \%$ & $53 \%$ \\
\hline Teen culture & 938 & 36,606 & $14 \%$ & $20 \%$ & $39 \%$ & $53 \%$ & $48 \%$ \\
\hline $\begin{array}{l}\text { Health and indi- } \\
\text { genous }\end{array}$ & 611 & 11,966 & $40 \%$ & $17 \%$ & $38 \%$ & $65 \%$ & $55 \%$ \\
\hline $\begin{array}{l}\text { Energy and envi- } \\
\text { ronment }\end{array}$ & 597 & 10,733 & $44 \%$ & $16 \%$ & $44 \%$ & $59 \%$ & $56 \%$ \\
\hline Science & 493 & 8,090 & $48 \%$ & $16 \%$ & $56 \%$ & $56 \%$ & $55 \%$ \\
\hline
\end{tabular}

Source: TrISMA (Bruns et al., 2016).

Towards the fringes of this agglomeration of political clusters are smaller, more topically focused groups, including energy and environment, health and indigenous, and science. Each used more hashtags and URLs in its tweets (between two-fifths and one-half of all their tweets contained hashtags); science in particular featured the two major hashtags \#SMP2017, for the Science Meets Parliament event in Canberra, and \#WSFB2017 (and variations), for the World Science Festival in Brisbane. Only 36 per cent of its tweets did not contain a hashtag. The overall science cluster, as detected by the Louvain algorithm, is subdivided into two communities in the Force Atlas 2 network visualization, representing these two distinct yet related events. 
Overall, we interpret the divergent hashtagging and link-sharing practices within these smaller topical clusters, and their positioning in the interactions network, as being indicative of a different, shorter-term nature in contrast to the larger, mainstream political clusters. The major political clusters represent politics as a longer-term theme; they are well-established public spherules (Bruns, 2008; Cunningham, 2001; Gitlin, 1998), the discussion networks of which have solidified into the clusters found in the follower network. They still use \#auspol to signify their thematic focus but no longer fundamentally rely on it to facilitate their connection. On any ordinary day, we expect to see these clusters in action much as we have observed them for 22 March 2017. The smaller specialist clusters, however, are issue publics (Dahlgren, 2009; Habermas, 2006) formed around short-term topics; they crucially rely on event- or issue-related hashtags to connect interactants, exist in part to share and amplify information on their issues through URL tweets and disperse again once the event concludes or the issue is resolved. Some users might even engage in multiple such publics at the same time and act as bridges between them. Many participants in these ad hoc publics (Bruns \& Burgess, 2015) may eventually return to the longer-term public spherules in which they usually engage and from which they temporarily departed to participate in the specific activities taking place on 22 March 2017. A conventional Habermasian model of "the" public sphere cannot capture these finer details and continues to relegate "networks for the wild flows of messages" such as social media to "the periphery of the political system" (Habermas, 2006: 415) - yet, when we focus on the everyday, mundane communicative practices of ordinary social media users, these networks, and the forms of publics that they support, are front and centre.

We suspect similar dynamics of transition between longer-term public spherules and shorter-term issue publics in the agglomeration of sports-related clusters towards the bottom of our interaction network. These clusters again mirror the long-term sports clusters found in the follower network: between AFL (Australian Football), NRL (Rugby League) and A-League (FIFA football), all the major football codes played in Australia are represented here, as are F1 motorsport and (further from mainstream sports) horse racing. However, while sports are clearly a permanent feature in the Twittersphere, and participant communities can be regarded as public spherules, such discussions are strongly dynamic as hashtag-driven ad hoc publics form around individual sporting events. We note issue publics beginning to form around hashtags such as \#F1 and \#AusGP ahead of the 2017 Australian Formula One Grand Prix in Melbourne on 26 March, and around the \#IRQvAUS match hashtag in anticipation of the FIFA World Cup qualifier in Tehran on 23 March. The following days would be likely to experience relevant clusters swelling further in size and activity before fading away as these events conclude.

Another major community in the Australian Twittersphere can be found in our interaction network: a teen culture cluster, surrounding the frequently mentioned accounts of One Direction and Five Seconds of Summer members and other stars, is located far from the politics clusters. While only 14 per cent of its tweets contained hashtags, those hashtags that were used here diverged strongly from the themes found elsewhere: hashtags such as \#dolantwinsnewvideo, \#funkykidsmusic and \#music4kids signal the cluster's thematic focus.

Situated in close proximity to this teen culture cluster is the largest cluster in the interaction network, comprising 2,440 accounts - which is also the most difficult to cat- 
egorize, however. Remarkably, 95 per cent of tweets by accounts in this cluster did not contain a hashtag; the 20 most @mentioned and retweeted accounts include pro skating legend Tony Hawk, various meme accounts, and a number of authors and creatives, as well as six accounts that have since been suspended by Twitter, two that are now private and one that has been discontinued for unknown reasons (at the time of writing, almost exactly one year later). Of the 20 most active accounts, 12 have been closed, another two suspended and one set to private, while the remainder show no obvious thematic focus. Some of these accounts have posted several tens of thousands of tweets during their time on Twitter but boast only a few hundred followers; therefore, they cannot be classed as microcelebrities (Senft, 2013). Several provide virtually no profile information. Finally, much of their activity on 22 March 2017 consisted of original tweets $(23 \%)$ and retweets (56\%), with comparatively few @mentions (25\%), so their focus appears to be on sharing personal updates or passing on the tweets of others. The number of suspensions might suggest that spam networks are driving the activity in this cluster, but the pronounced absence of hashtags contradicts that hypothesis, since hashtags are commonly used by spammers to enhance the visibility of their posts. Rather, we interpret the accounts in this cluster as engaging in genuine phatic sharing and have named the cluster accordingly.

We acknowledge the shortcomings of this term. It imposes a single descriptor on what is in reality a collection of diverse but related practices. Phatic sharing is an example of what earlier researchers might have labelled "banal" or Pear Analytics classed as "pointless babble" (2009: 4), but such disparaging terms have rightly been rejected as valorizing only a narrow range of activities. An alternative label could be "shitposting" (McEwan, 2017), but that term increasingly appears to be associated with deliberate attempts, especially by far-right trolls, to derail meaningful discussions by posting offensive and derogatory content (Griffin, 2016) and no longer maintains its earlier, more neutral meaning of "posting stupid things". We therefore propose phatic sharing as a provisional term for these mundane, everyday practices of posting not-quite-random content, or retweeting the phatic content, of others to present an online persona and maintain a connection to the network beyond. We suggest that further work should identify a range of distinct phatic sharing practices.

Together, phatic sharing and teen culture represent Twitter uses that are very different from the practices in the political and sporting clusters: their emphasis is on signalling one's existence as a Twitter participant by providing a stream of personal content (original tweets) and shared updates (retweets). This combines, in the teen culture cluster, with frequent professions of fandom for various music and movie celebrities (through @ mentions of their accounts). Such activity may be influenced by external events - the release of new music or movies - and manifest in relevant hashtags, but we expect it to persist fairly steadily over time; these clusters are likely to represent comparatively stable public spherules rather than short-lived issue publics. This is documented for teen culture by its substantial presence on the follower network map, but (because of that map's focus on accounts with at least 1,000 follower/followee connections) not as clearly for phatic sharing, in which many active participants do not reach that threshold.

The hourly activity patterns in the ten major clusters support these interpretations (Figure 5). Phatic sharing rose to an early peak after 9:00 and then declined towards the afternoon before reaching a new peak in the after-dinner hours; this suits casual 
Figure 5. Tweeting activity by accounts in ten major interactivity network clusters over the course of the day

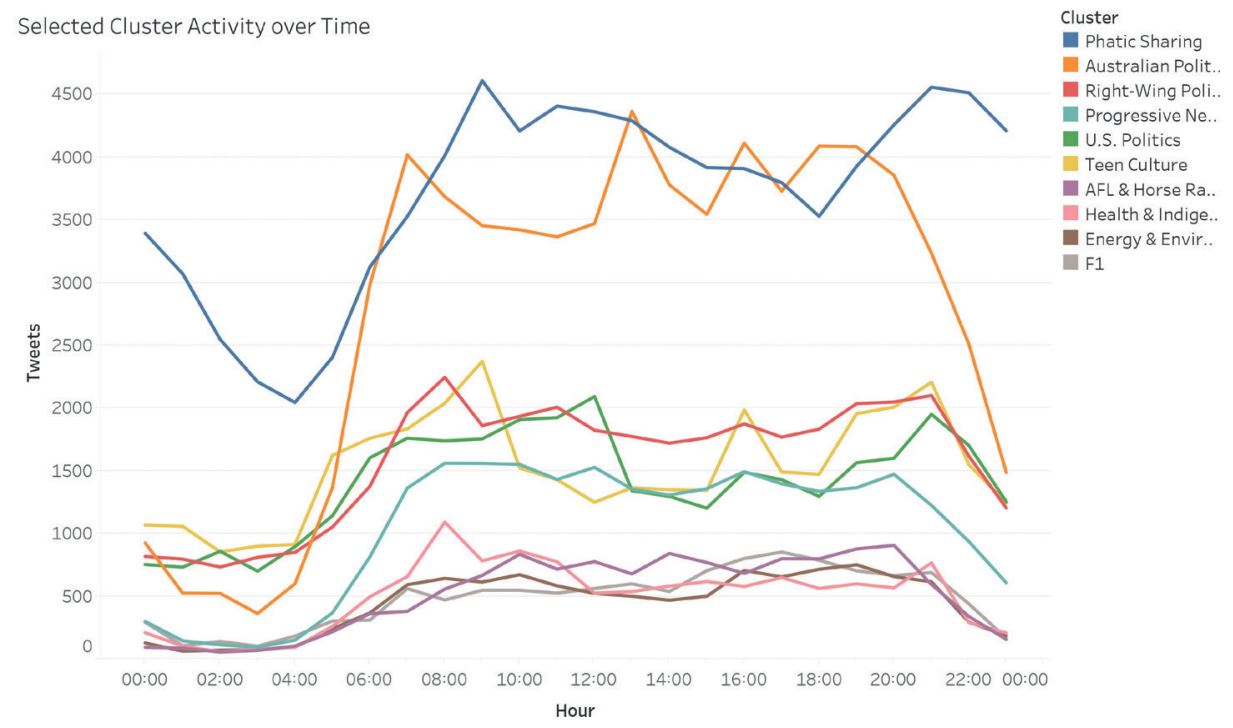

Source: TrISMA (Bruns et al., 2016).

practice during off-peak times at work or at home. Teen culture showed a similar early peak, perhaps representing students heading to school, and a minor peak after 16:00, which could signify activity on the journey home, and more engagement in the evening but declining after 21:00. Meanwhile, most politics clusters remained fairly steady throughout the day, except for a pronounced spike in Australian politics after 13:00 as Question Time unfolded. US politics, by contrast, was prominent during the morning but less so in the afternoon, probably because 13:00 AEST equates to 23:00 EDT; the activity returned later in the day, as a new day began in the United States.

\section{Conclusion and outlook}

In this article, we have provided only a broad overview of activity patterns for only a single 24-hour period. However, several important observations can already be made. First, as suspected, there is considerably greater diversity of tweeting within this national Twittersphere than conventional hashtag studies have captured; in particular, the highly active yet extraordinarily low-key and indistinct phatic sharing cluster represents a collection of accounts and tweeting practices that to date have failed to register in other studies. Such practices may contribute a sizeable component to the global volume of tweets; our observations offer merely a glimpse of the constant activity of ordinary, everyday Twitter users with no interest in news or politics on the platform. The methodological difficulties inherent in capturing the intrinsically unremarkable have left these practices severely underresearched.

This addresses our second research question: the alternative approach sketched out here can reveal previously overlooked but potentially widespread user practices. As Table 2 shows, phatic sharers and their tweets clearly could not be investigated using 
standard hashtag-driven methods, as only around five per cent of their tweets contain hashtags; further, given the diversity and idiosyncrasy of what they tweet and retweet, even keyword-based collection approaches would struggle to capture more than a small and unrepresentative subset of their content. Similarly, network-based methods that begin with the most prominent accounts in the Twittersphere overlook this loosely organized community of phatic sharers, such as Bruns and colleagues (2017) with their focus on accounts with at least 1,000 global follower/followee connections. Phatic sharing is prominent in the Australian Twittersphere but occurs predominantly in the shadows of the long tail rather than around those accounts that are exposed to the light by their large follower bases or that actively seek visibility by engaging with leading hashtags.

Phatic sharing is therefore emblematic of the mundane, everyday experiences of ordinary users: individually, they rarely generate a significant impact, by any conventional metric, but collectively they represent a leading use of Twitter - yet that use remains almost entirely unrecognized by standard data-gathering methods that centre on the loudest voices (hashtags) or greatest stars (followers). Only a whole-of-population approach, as implemented by TrISMA or enabled by the global Twitter firehose, can illuminate these hidden practices, at scale, by separating out known communities and examining what remains, and even then only if we approach these data sets without preconceived notions of what is "banal" and what is not.

Second, in comparison with the longer-term follower/followee network, the shortterm interaction patterns during our 24-hour window facilitate a tentative assessment of how these two network layers intersect. This answers our third research question: our observations support the view that comparatively stable public spherules on persistent themes in politics, sports and culture, in which the structures of follower and interaction networks mirror each other, coexist with shorter-lived issue publics that emerge around particular topics and events and that bring together previously unconnected accounts. Within these distinct communities of participants, diverging practices of using the affordances of the platform (@mentions, retweets, hashtags, embedded URLs, etc.) emerge in accordance with the interests of the community. This dynamic process of transition between issue publics and public spherules deserves more attention: for instance, under what conditions might ad hoc issue publics solidify into persistent public spherules? An empirical examination of such diachronic processes requires far more than a 24-hour data set, but even our study of a single, comparatively ordinary day may have captured such a process at an intermediate stage, as ad hoc discussions about the Trump presidency solidified into a longer-term community examining collusion between the Trump campaign and its foreign enablers. These dynamics speak in important ways to the wider question of extracting broader meaning from onlife traces: the solidification of short-term issue publics into longer-term public spherules on Twitter is likely to mirror the crystallization of the interpretive frames through which society at large approaches a given topic, even if the specific demographics of Twitter may result in divergent sets of frames.

Finally, this article also demonstrates the opportunities and difficulties involved in working with "big social data" from leading social media platforms as we pursue the everyday, mundane onlife traces embedded in ordinary users' activities. To address the first research question, we deliberately pursued a mixed-method approach that combined initial time series, statistical and network analysis with extensive close reading 
and qualitative interpretation. This abductive approach (Dixon, 2012) is appropriate at a stage of research that focuses on the formation rather than the testing of hypotheses. Such interpretation requires a solid understanding of what may be contained in the data, however, and cannot easily be replicated at a much larger, possibly transnational, multilingual scale. A purely computational, quantitative approach to the data set might have overlooked some finer details in the data or dismissed the amorphous phatic sharing cluster simply as "pointless babble". It remains necessary to develop, test and apply new methods for capturing more than is already known about Twitter users' practices, focusing especially on the everyday and apparently unremarkable; to examine and interpret such practices through a combined quantitative and qualitative perspective that allows for the unexpected; and finally to channel these observations into testable hypotheses about the longer-term dynamics of Twitter use. We are still a considerable way from reliably extracting meaning from these observational onlife traces, but this incremental, explorative, abductive process, drawing on mixed methods for the analysis of complex data sets, is likely to be critical as we proceed.

\section{Funding}

This research was supported by the Australian Research Council through the ARC Future Fellowship project Understanding intermedia information flows in the Australian online public sphere [FT130100703] and the ARC LIEF project TrISMA: Tracking infrastructure for social media analysis [LE140100148].

\section{References}

Blaszka, M., Burch, L. M., Frederick, E. L., Clavio, G. \& Walsh, P. (2012). \#WorldSeries: An empirical examination of a Twitter hashtag during a major sporting event. International Journal of Sport Communication, 5(4): 435-453.

Blondel, V. D., Guillaume, J.-L., Lambiotte, R. \& Lefebvre, E. (2008). Fast unfolding of communities in large networks. Journal of Statistical Mechanics: Theory and Experiment, 10: P10008. doi: http://doi. org/10.1088/1742-5468/2008/10/P10008

Bruns, A. (2008). Life beyond the public sphere: Towards a networked model for political deliberation. Information Polity, 13(1-2): 71-85.

Bruns, A. \& Burgess, J. (2015). Twitter hashtags from ad hoc to calculated publics. In N. Rambukkana (ed.), Hashtag publics: The power and politics of discursive networks (pp. 13-28). New York: Peter Lang.

Bruns, A. \& Moe, H. (2014). Structural layers of communication on Twitter. In K. Weller, A. Bruns, J. Burgess, M. Mahrt \& C. Puschmann (eds.), Twitter and society (pp. 15-28). New York: Peter Lang.

Bruns, A., Burgess, J., Banks, J., Tjondronegoro, D., Dreiling, A., Hartley, J., Leaver, T., Aly, A., Highfield, T., Wilken, R., Rennie, E., Lusher, D., Allen, M., Marshall, D., Demetrious, K. \& Sadkowsky, T. (2016). TrISMA: Tracking infrastructure for social media analysis. Retrieved from http://trisma.org/ [Accessed 2018, December 7].

Bruns, A., Moon, B., Münch, F. \& Sadkowsky, T. (2017). The Australian Twittersphere in 2016: Mapping the follower/followee network. Social Media + Society, 3(4): 1-15. doi: http://doi. org/10.1177/2056305117748162.

Burgess, J. \& Bruns, A. (2015). Easy data, hard data: The politics and pragmatics of Twitter research after the computational turn. In G. Langlois, J. Redden \& G. Elmer (eds.), Compromised data: From social media to big data (pp. 94-111). New York: Bloomsbury Academic.

Coleman, S. (2003). A tale of two houses: The House of Commons, the Big brother house and the people at home. Parliamentary Affairs, 56: 733-758. doi: http://doi.org/10.1093/pa/gsg113.

Crawford, K. (2009). Following you: Disciplines of listening in social media. Continuum, 23(4): 525-535. doi: http://doi.org/10.1080/10304310903003270

Cunningham, S. (2001). Popular media as public "sphericules" for diasporic communities. International Journal of Cultural Studies, 4(2): 131-147. doi: http://doi.org10.1177/136787790100400201 
Dahlgren, P. (2009). Media and political engagement: Citizens, communication, and democracy. Cambridge: Cambridge University Press.

Dixon, D. (2012). Analysis tool or research methodology? Is there an epistemology for patterns? In D. M. Berry (ed.), Understanding digital humanities (pp. 191-209). Houndmills, UK: Palgrave Macmillan.

Gitlin, T. (1998). Public sphere or public sphericules? In J. Curran \& T. Liebes (eds.), Media, ritual and identity (pp. 175-202). London: Routledge.

Griffin, A. (2016, September 23). Shitposting: What is the bizarre online behaviour that could win Donald Trump the election? The Independent [online]. Retrieved from https:/www.independent.co.uk/life-style/ gadgets-and-tech/news/what-is-shitposting-donald-trump-us-election-2016-palmer-luckey-a7326111. html [Accessed 2018, December 7].

Habermas, J. (2006). Political communication in media society: Does democracy still enjoy an epistemic dimension? The impact of normative theory on empirical research. Communication Theory, 16: 411-426. doi: http://doi.org/10.1111/j.1468-2885.2006.00280.x.

Haddon, L. (2016). The domestication of complex media repertoires. In K. Sandvik, A. M. Thorhauge \& B. Valtysson (eds.), The media and the mundane: Communication across media in everyday life (pp. 1729). Gothenburg: Nordicom.

Highfield, T. (2013). National and state-level politics on social media: Twitter, Australian political discussions, and the online commentariat. International Journal of Electronic Governance, 6(4): 342-360. doi: http:// doi.org/10.1504/IJEG.2013.060648.

Highfield, T. (2014). Following the yellow jersey: Tweeting the Tour de France. In K. Weller, A. Bruns, J. Burgess, M. Mahrt \& C. Puschmann (eds.), Twitter and society (pp. 249-262). New York: Peter Lang.

Jacomy, M., Venturini, T., Heymann, S. \& Bastian, M. (2014). ForceAtlas2, a continuous graph layout algorithm for handy network visualization designed for the Gephi software. PLOS ONE, 9(6) doi: http://doi. org/10.1371/journal.pone.0098679

Larsson, A. O. \& Moe, H. (2014). Twitter in politics and elections: Insights from Scandinavia. In K. Weller, A. Bruns, J. Burgess, M. Mahrt \& C. Puschmann (eds.), Twitter and society (pp. 319-330). New York: Peter Lang.

Marwick, A. E. \& boyd, danah (2011). I tweet honestly, I tweet passionately: Twitter users, context collapse, and the imagined audience. New Media \& Society, 13(1): 114-133. doi: http://doi. org/10.1177/1461444810365313.

McEwan, S. (2017). Nation of shitposters: Ironic engagement with the Facebook posts of Shannon Noll as reconfiguration of an Australian national identity. Journal of Media and Communication, 8(2): 19-39.

McKinnon, M., Semmens, D., Moon, B., Amarasekara, I. \& Bolliet, L. (2016). Science, Twitter and election campaigns: Tracking \#auspol in the Australian federal elections. Journal of Science Communication, 15(6). doi: http://doi.org/10.22323/2.15060204.

McNamara, L. (2016, September 1). Explainer: What is Section 18C and why do some politicians want it changed? The Conversation [online]. Retrieved from https://theconversation.com/explainer-what-issection-18c-and-why-do-some-politicians-want-it-changed-64660 [Accessed 2018, December 7].

Münch, F. (2019). Measuring networked media - Exploring network science methods for large scale online media studies. PhD Dissertation. Brisbane: Queensland University of Technology.

Murthy, D. (2018). Twitter (2nd ed.). Cambridge: Polity.

Palen, L., Starbird, K., Vieweg, S. \& Hughes, A. (2010). Twitter-based information distribution during the 2009 Red River Valley flood threat. Bulletin of the American Society for Information Science and Technology, 36(5): 13-17.

Papacharissi, Z. \& Blasiola, S. (2016). Structures of feeling, storytelling, and social media: The case of \#Egypt. In A. Bruns, G. Enli, E. Skogerbø, A. O. Larsson \& C. Christensen (eds.), The Routledge companion to social media and politics (pp. 211-222). London: Routledge.

Pariser, E. (2011). The filter bubble: What the Internet is hiding from you. London: Penguin.

Pear Analytics. (2009). Twitter study-August 2009. Retrieved from http://www.pearanalytics.com/wp-content/ uploads/2012/12/Twitter-Study-August-2009.pdf [Accessed 2018, December 7].

Rambukkana, N. (ed.). (2015). Hashtag publics: The power and politics of discursive networks. New York: Peter Lang.

Rogers, R. (2014). Foreword: Debanalising Twitter: The transformation of an object of study. In K. Weller, A. Bruns, J. Burgess, M. Mahrt \& C. Puschmann (eds.), Twitter and society (pp. ix-xxvi). New York: Peter Lang.

Sandvik, K., Thorhauge, A. M. \& Valtysson, B. (2016). Introduction. In K. Sandvik, A. M. Thorhauge \& B. Valtysson (eds.), The media and the mundane: Communication across media in everyday life (pp. 9-15). Gothenburg: Nordicom.

Senft, T. M. (2013). Microcelebrity and the branded self. In J. Hartley, J. Burgess \& A. Bruns (eds.), A companion to new media dynamics (pp. 346-354). London: Blackwell. 
Sensis. (2016). Sensis social media report 2016: How Australian people and businesses are using social media. Melbourne: Sensis. Retrieved from https://www.sensis.com.au/asset/PDFdirectory/Sensis Social_Media_Report_2016.PDF[Accessed 2018, December 7].

Shaw, F., Burgess, J., Crawford, K. \& Bruns, A. (2013). Sharing news, making sense, saying thanks: Patterns of talk on Twitter during the Queensland floods. Australian Journal of Communication, 40(1): 23-39.

Simon, J. \& Ess, C. (2015). Philosophy \& Technology, 28(1): 157-162. doi: http://doi.org/10.1007/s13347015-0189-8.

Sunstein, C. R. (2009). Republic.com 2.0. Princeton, NJ: Princeton University Press.

Van Dijck, J. \& Poell, T. (2013). Understanding social media logic. Media and Communication, 1(1): 2-14. doi: http://doi.org.10.12924/mac2013.01010002. 